\title{
The James Webb Space Telescope: Mission Overview and Status
}

\author{
Matthew Greenhouse and The JWST Science Working Group ${ }^{1}$
}

\begin{abstract}
The James Webb Space Telescope (JWST) is the infrared successor to the Hubble Space Telescope. It is a cryogenic infrared space observatory with a $25 \mathrm{~m}^{2}$ aperture (6 m class) telescope yielding diffraction limited angular resolution at a wavelength of $2 \mathrm{um}$. The science instrument payload includes three passively cooled near-infrared instruments providing broad- and narrow-band imagery, coronagraphy, as well as multi-object and integral-field spectroscopy over the $0.6<\square<5.0 \mathrm{Dm} \mathrm{spec}$ trum. An actively cooled mid-infrared instrument provides broad-band imagery, coronagraphy, and integral-field spectroscopy over the $5.0<\square<29 \square \mathrm{m}$ spectrum. The JWST is being developed by NASA, in partnership with the European and Canadian Space Agencies, as a general user facility with science observations to be proposed by the international astronomical community in a manner similar to the Hubble Space Telescope. Technology development and mission design are complete, and construction is underway in all areas of the program. The JWST is on schedule to reach launch readiness during 2014.
\end{abstract}

\section{Designing for Discovery}

The science motivation for the JWST mission was developed by a succession of international science working groups and is described by Gardner et al. 2006, with updates maintained by the JWST flight science working group ${ }^{1}$ at: http://www.stsci.edu/jwst/science/whitepapers/. This science case forms the basis from which detailed science and mission requirements were derived to guide engineering design and development of the JWST as a research tool. The science observations that are actually implemented by the JWST will be proposed by the international astronomical community in response to annual peer reviewed proposal opportunities. The discovery potential of the JWST relative to other concurrent facilities is discussed in Thronson, Stiavelli, and Tielens 2009.

The emergence of the first sources of light in the universe (after decoupling) marks the end of the "Dark Ages" in cosmic history (Rees 1997). The ultraviolet radiation field produced by these sources created the ionization that is observed in the local intergalactic medium (IGM). The JWST design provides unique capability to address key questions about this era in cosmic evolution including: what is the nature of the first galaxies; how and when did ionization of the space between them occur; and what sources caused the ionization?

The JWST architecture is primarily shaped by requirements associated with answering the above questions. In contrast to the Hubble Space Telescope (HST), the JWST is designed as an infrared optimized telescope to observe the red shifted ultraviolet radiation from the first galaxies and supernovae of the first stars. To achieve the nJy sensitivity needed to observe this era $(z \sim 6-20)$, the observatory must have a telescope aperture that is larger in diameter than the largest rocket faring, and the entire optical system must be cooled to $\sim 40-50 \mathrm{~K}$. Finally, the resulting large deployable cryogenic telescope must achieve HST-like angular resolution across the SWIR spectrum. The major observatory design features seen in Figure 1 trace directly from these requirements and differ markedly from those of the HST (Table 1).

${ }^{1}$ John C. Mather, Mark Clampin, Rene Doyon, Kathryn A. Flanagan, Marijn Franx, Matthew A. Greenhouse, Heidi B. Hammel, John B. Hutchings, Peter Jakobsen, Simon J. Lilly, Jonathan I. Lunine, Mark J. McCaughrean, Matt Mountain, George H. Rieke, Marcia J. Rieke, George Sonneborn, Massimo Stiavelli, Rogier Windhorst and Gillian S. Wright. 


\begin{tabular}{|l|c|c|}
\hline \multicolumn{1}{|c|}{ Performance Parameter } & HST & JWST \\
\hline OTE Diameter (meters) & 2.4 & 6.1 by 6.6 \\
\hline Mass (kg) & 11600 & 6330 \\
\hline Output Power at Load Input (watts) & 2400 & 2079 \\
\hline Unobscured Aperture (Sq meters) & 4.5 & 25 \\
\hline Overall Optical Transmission & 45 to $25 \%$ & $62 \%$ to $43 \%$ \\
\hline Telescope Field of View (Arcmin) & 14.6 (Radius) & 18 by 9 \\
\hline Wavelength of Diffraction Limited Performance (Microns) & 0.5 & 2 \\
\hline Rayliegh Radius (Arcsec) & 0.043 & 0.069 \\
\hline Telescope Strehl Ratio & $80 \%$ & $80 \%$ \\
\hline Pointing Accuracywithout Fine Guidance (Arcsec) & 22 & 7 \\
\hline Pointing Stability with Fine Guidance (Arcsec) & 0.007 & 0.007 \\
\hline Total Pixels (Megapixels) & 25 & 60 \\
\hline Data Throughput (Gbits/day) & 27 & 471 \\
\hline Observing Efficiency & $50 \%$ & $70 \%$ \\
\hline
\end{tabular}

Table 1: Key design parameters of the JWST and HST

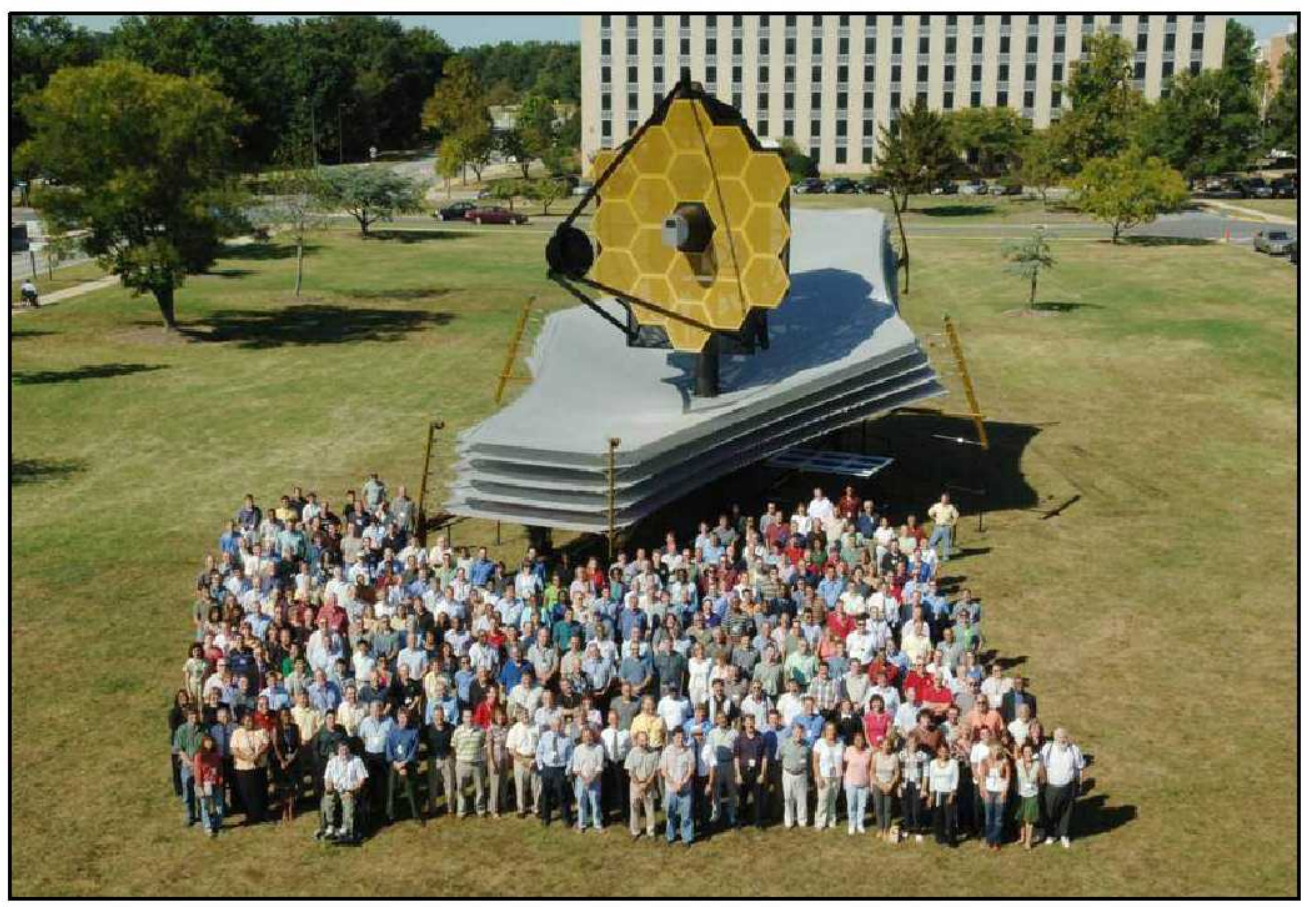

Figure 1: Full scale display model of the JWST space vehicle

The JWST science instrument payload is designed to probe the first galaxies with high angular resolution near-infrared image surveys in broad-band filters. This capability enables identification of primeval galaxies by searching their Lyman continuum break with multi-filter photometry. This prominent feature occurs in the near-infrared $(1.3-2.6 \mathrm{um})$ for galaxies with redshifts in the range of $10-20$. This broad-band technique exploits the maximum sensitivity of the observatory, such that the space density of galaxies can be probed to z 20. JWST high angular resolution imagery across the $0.6-29$ um spectrum will probe the assembly and evolution of galaxy morphologies to enable observation of when and how the Hubble sequence formed. 
The JWST is designed to enable near-infrared multi-object spectroscopy of thousands of galaxies at several spectral resolutions $\left(\sim 10^{2}, \sim 10^{3}\right)$. This capability will probe the chemical evolution and metallicity of galaxies, and the ionization state of the IGM across cosmic time. Low resolution multi-object spectroscopy will enable calibration of photometric redshifts for primeval galaxy studies. The JWST spectrometers include integral field capability over the $0.6-29$ um spectrum that will enable detailed spectral, morphological, and kinematic studies of high redshift galaxies and local galaxy nuclei. JWST spectroscopy includes wide field scanning Fabry-Perot and narrow-band filter imagery at low $(\sim 1 \%)$ spectral resolution and high angular resolution to enable both wide field emission line imagery and high redshift surveys of emission line galaxies.

The JWST observatory design enables wide discovery potential beyond cosmology and galaxy studies. The JWST high angular resolution imagery and imaging spectroscopy across the $0.6-29$ um spectrum will open a new window on observation of star formation in our own galaxy to reveal: how molecular clouds collapse; how environment effects star formation and vice-versa; the mass distribution of low mass stars; and the relationship between stellar debris disks the formation of terrestrial planets.

The JWST instruments include coronagraphic imagery and spectroscopy capability that will enable a wide range of stellar debris disk studies and extra-solar planet observations at high angular resolution. High dynamic range modes of the JWST instruments will enable extra-solar planet transit photometry and spectroscopy across the above wavelength range. The JWST observatory enables non-sidereal tracking so that the full observatory capability can be used observe the outer solar system to enable comparative studies between stellar debris disks and our own solar system.

\section{Architecting for success}

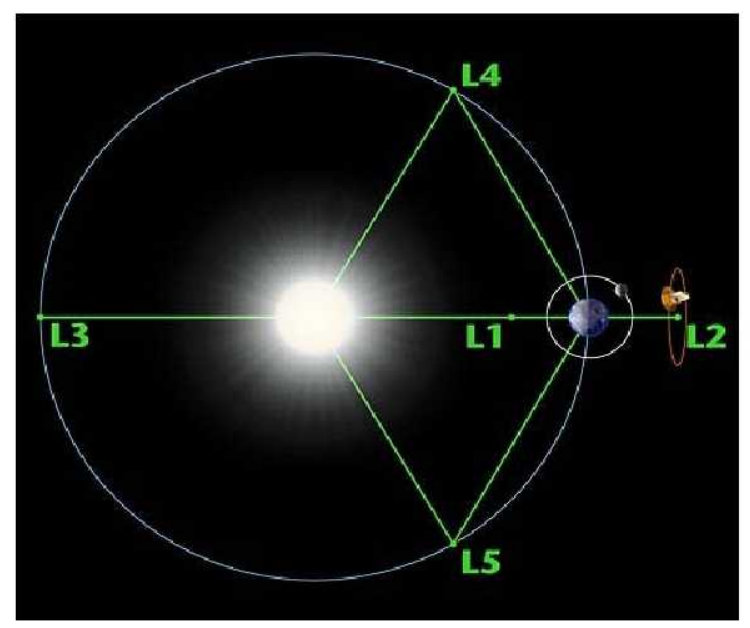

Figure 2: The JWST will orbit the Sun-Earth L2 point.

The large primary mirror area and cryogenic operating temperature are key drivers on the JWST mission architecture. The telescope is the largest space telescope ever constructed. Liquid cryogen cooling techniques used by prior infrared space observatories (IRAS, ISO, Spitzer) cannot be practically scaled to meet JWST requirements, and existing cryo-cooler technology could not meet the heat lift requirements of this large system. As a consequence, a passively cooled architecture was adopted for the majority of the system. A libration point orbit about the Sun-Earth L2 point (Figure 2) was selected to meet this requirement. In this orbit, the observatory follows the earth around the sun such that the sun and earth always appear in the same direction. Hence, it is possible to continuously shield portions of the observatory from the sun and earth to enable passive cryogenic cooling. The orbit about the L2 point itself is sized to avoid eclipses; thus enabling continuous generation of power with solar arrays. This orbit has a period of approximately 6 months and is unstable requiring use of station keeping thrusters. Propellant for orbit maintenance and momentum management ultimately limits the lifetime of the observatory to approximately twice the duration of its 5 year science mission.

The JWST can observe the whole sky while remaining continuously in the shadow of its sun shield. As shown in Figure 3, the space vehicle can pitch through an angle of 50 degrees and rotate completely about the earth-sun line to observe sources within an annulus that covers approximately $39 \%$ of the sky. As the observatory orbits the sun, this annulus sweeps over the whole sky each year with small continuous viewing zones at the ecliptic poles. 
The JWST can reach this orbit in approximately 100 days after launch from Kourou Launch Center in French Guiana using an Arian 5 ECA launch vehicle via a direct transfer trajectory. This class of launch vehicle provides adequate fairing volume and mass capability to enable an observatory design that meets the above science requirements.

The $6.5 \mathrm{~m}$ diameter telescope and its tennis court sized sunshield, are designed to be stowed within the Arian $5 \mathrm{~m}$ diameter faring along with the science instrument payload and spacecraft (Figures 4, 5) such that the observatory can deploy into the operational configuration shown in Figure 1 (see animation at: www.jwst.nasa.gov/videos_deploy.h $\mathrm{tml})$. The observatory is launched warm and cooling begins after sunshield deployment in route to the L2 point.
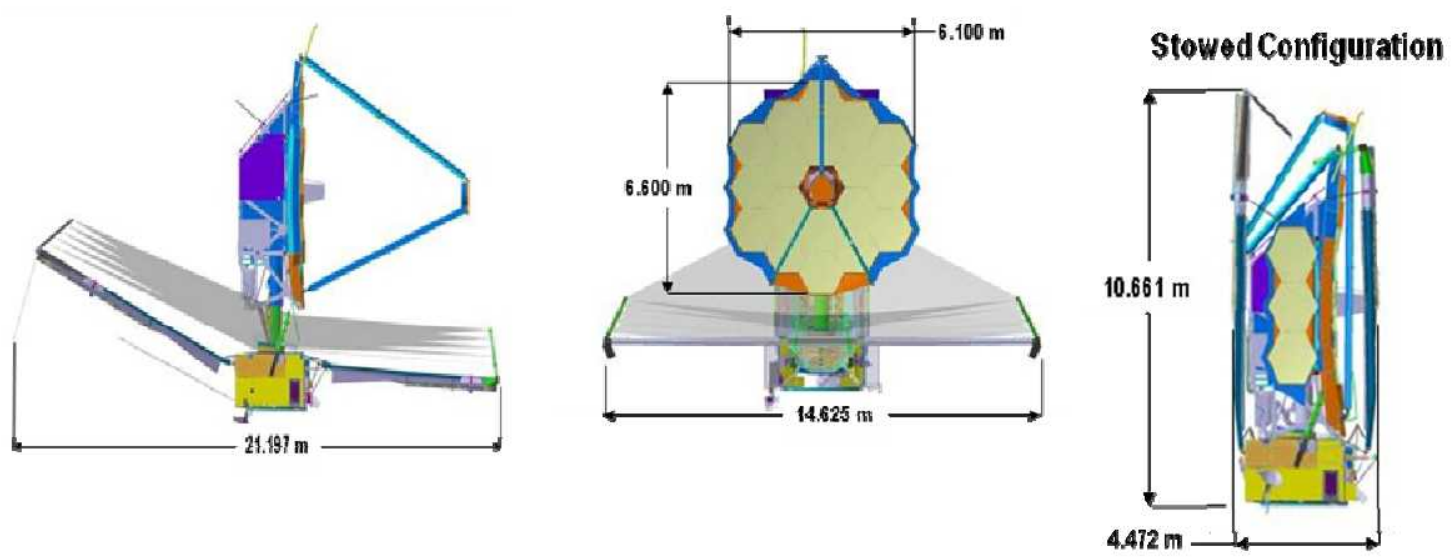

Figure 4: The JWST is the largest cryogenic optical system ever constructed 

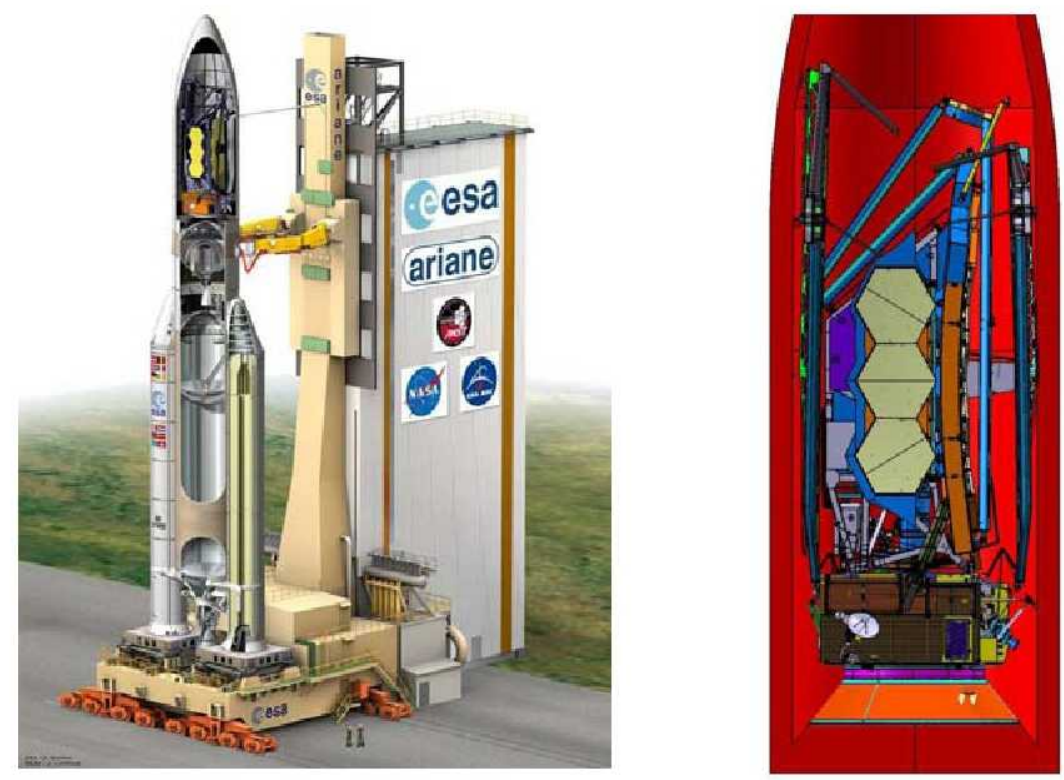

Figure 5: The JWST is designed to integrate with an Arian $5 \mathrm{~m}$ diameter faring

The resulting mission architecture is shown in Figure 6. During operations, the NASA Deep Space Network is used to support two $4 \mathrm{hr}$ communications contacts each day during which approximately 470 Gbits of science and engineering data are downloaded. Both mission and science operations are supported by the Space Telescope Science Institute. Overall mission management, as well as guidance navigation and control, are provided by Goddard Space Flight Center.

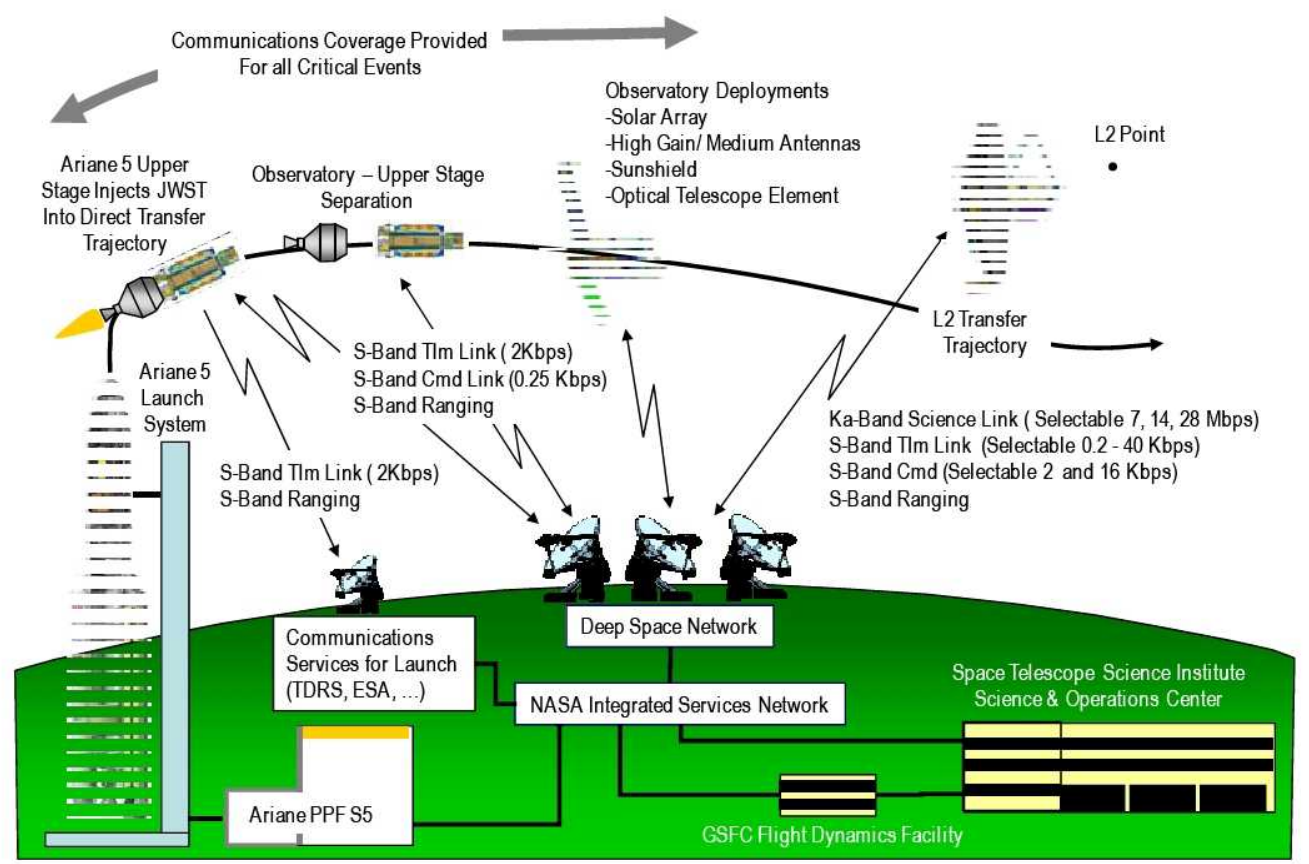

Figure 6: The JWST mission architecture 


\section{Collecting the first light}

A three mirror anastigmat (TMA) telescope design was selected to enable high image quality over a wide field of view. In contrast to ground-based telescopes, minimizing mass is a key design driver on the JWST telescope. Beryllium was selected as a material for the three TMA mirrors due to its high thermal conductivity, high stiffness to mass ratio, and low expansion coefficient at the $\sim 50 \mathrm{~K}$ operating temperature. A segmented primary mirror design was chosen to enable fold-up stowage as shown in Figure 7. The deployed aperture is tricontagon in shape with a collecting area of $25 \mathrm{~m}^{2}$. The primary mirror is composed of 18 hexagonal segments $1.32 \mathrm{~m}$ flat-to-flat of three optical prescriptions. Each primary mirror segment and the secondary mirror are mechanized to provide in-flight position adjustment in 6 degrees of freedom (Figure 8). The primary mirror segments also have in-flight radius of curvature adjustment. A fine steering mirror is located near a pupil position (Figure 7). This mirror is servo controlled using an image-based fine guidance sensor located in the science instrument focal plane to enable 7 mas rms pointing stability. The mirrors are polished to a cryogenic figure error of approximately $20 \mathrm{~nm}$ rms via an iterative cryo-polishing process. Gold was selected as a mirror coating to provide high throughput over the 0.6 - 29 micron spectrum. This coating choice limits the JWST to wavelengths $>0.6 \mathrm{um}$. The $29 \mathrm{um}$ long wavelength limit results from detector technology and cooling constraints. The telescope structure consists of a M55J-954-6 cynate ester composite material that affords a high stiffness to mass ratio and a low cryogenic expansion coefficient to yield high optical alignment stability.
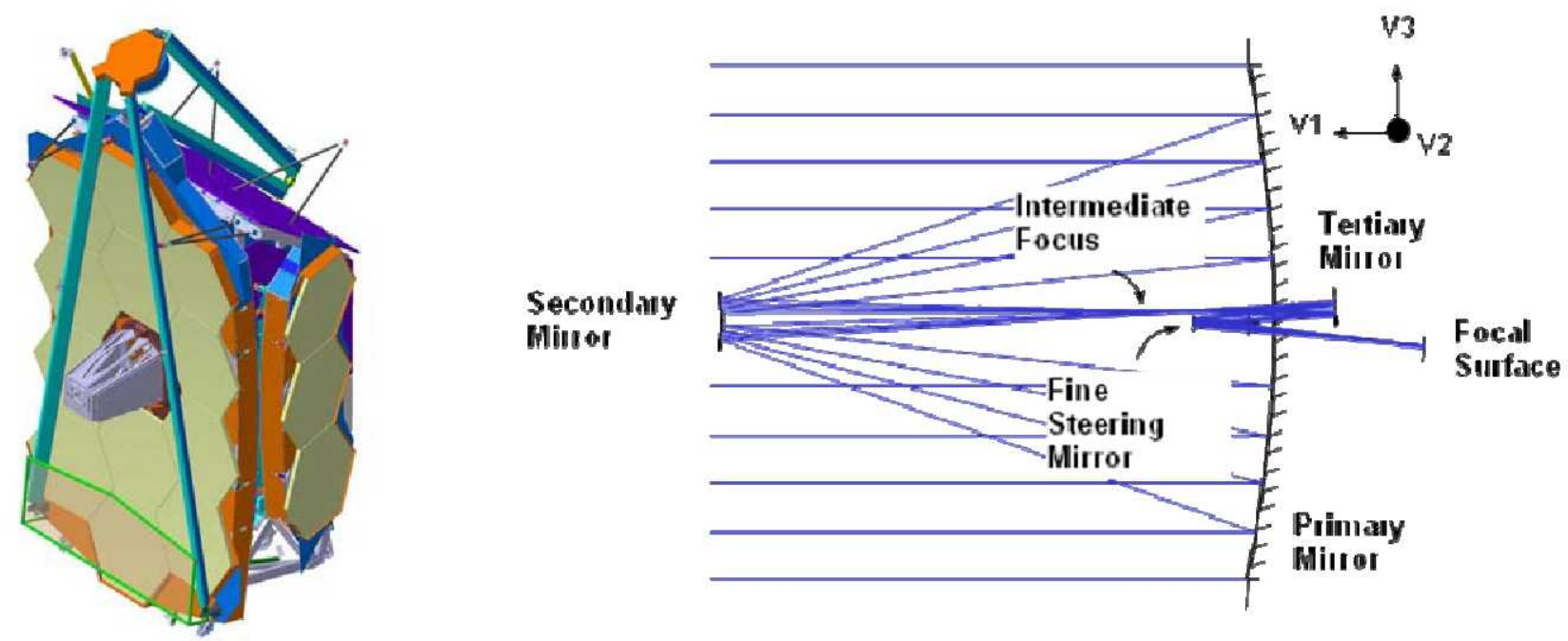

Figure 7: Left - The telescope in it stowed configuration; Right -- The telescope deploys into a three mirror anastigmat configuration
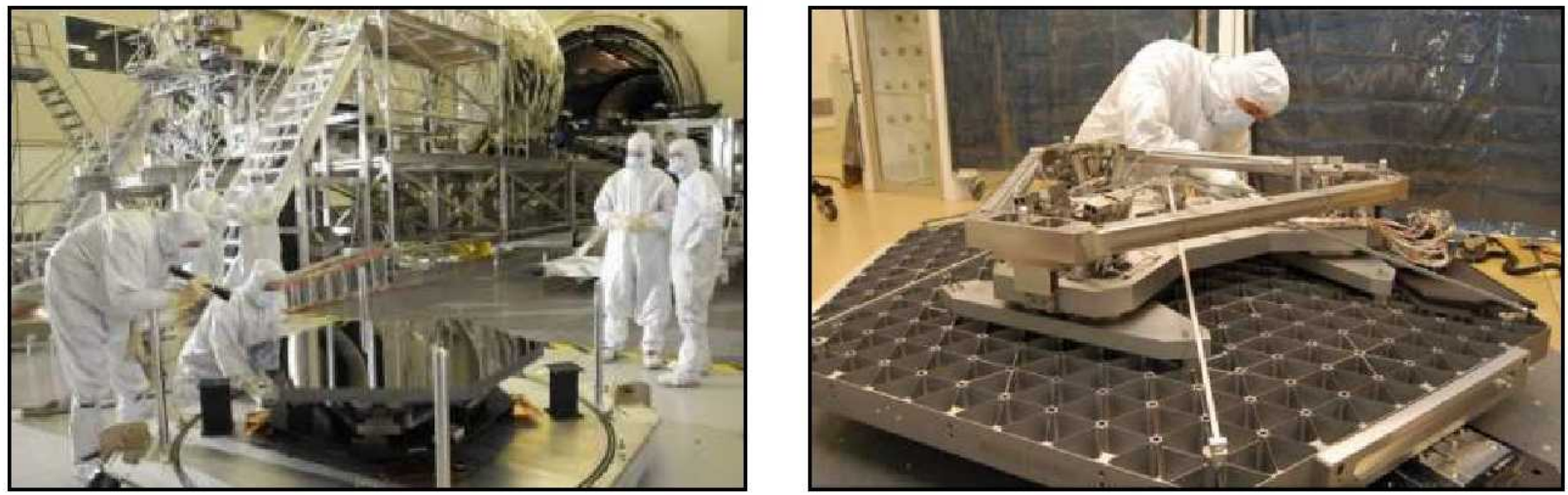

Figure 8: A primary mirror segment entering cryogenic testing (left), mechanized segment mount assembly provides 6 DOF and radius of curvature adjustment (right) 

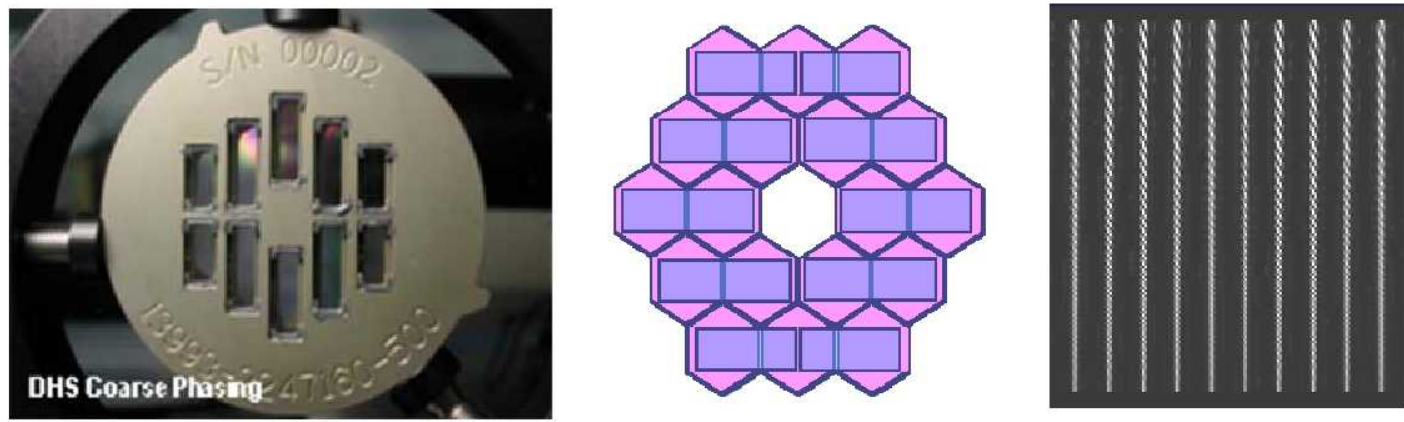

Figure 10: Dispersed Hartman sensing is used to perform coarse phasing of the mirror segments

The mirror segment actuators are periodically adjusted in flight to ensure diffraction limited image quality throughout the mission. The observatory's main near-infrared science camera (Section 4) is used as the wavefront error sensor for this process. Initial coarse phasing of the segments is accomplished using dispersed Hartman sensing optics in this camera (Figure 9). The subsequent fine phasing is performed on defocused images using a modified Gerchberg-Saxton algorithm (Acton 2004). This process is designed to achieve a telescope Strehl ratio of 0.8 at a wavelength of $2 \mathrm{um}$. The dispersed Hartman coarse phasing process was demonstrated on the Keck telescope (Albanese 2006). The overall flight wavefront sensing and control process has been demonstrated on a $1 / 6$ scale fully functional model (Figure 10) of the JWST telescope (Fein-

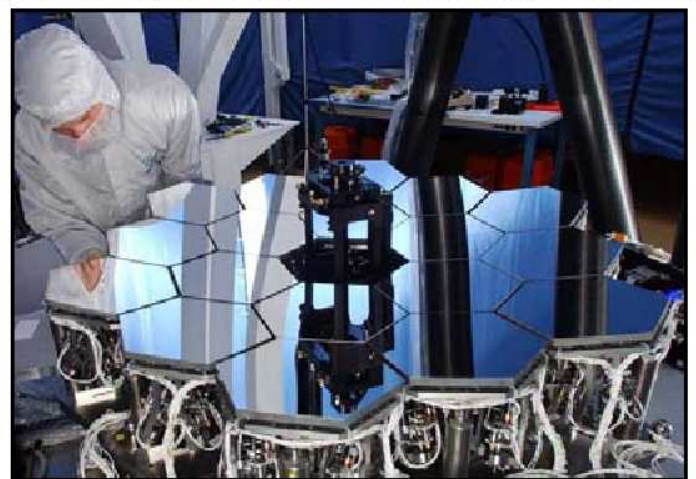

Figure 9: Fully functional 1/6 scale telescope model used to develop wavefront sensing and control process and algorithms berg 2007, Contos 2008).

\section{Extracting information from starlight}

The JWST science instrument payload contains four science instruments, a fine guidance sensor, and supporting systems for instrument control, command and data handling, cryogenic thermal control, and other functions (Greenhouse 2006). Near-infrared imagery is provided by the NIRCam instrument (Rieke 2005, 2008) shown in Figure 10. This camera provides high angular resolution wide-field imagery over the $0.6-5 \mathrm{um}$ spectrum. The detector pixel scale is chosen to optimally sample the telescope point spread function across this wavelength range by use of a dichroic beam splitter (Table 2). Two identical optical modules image adjacent fields of approximately 4 square arcminuets to provide full redundancy for telescope wavefront sensing. Occulting coronagraphy, yielding a rejection ratio of $\sim 10^{4}$, is provided in both long and short wavelength channels. All focal plane arrays support high cadence sub-array exposures to provide a high dynamic range capability for exoplanet transit observations. 

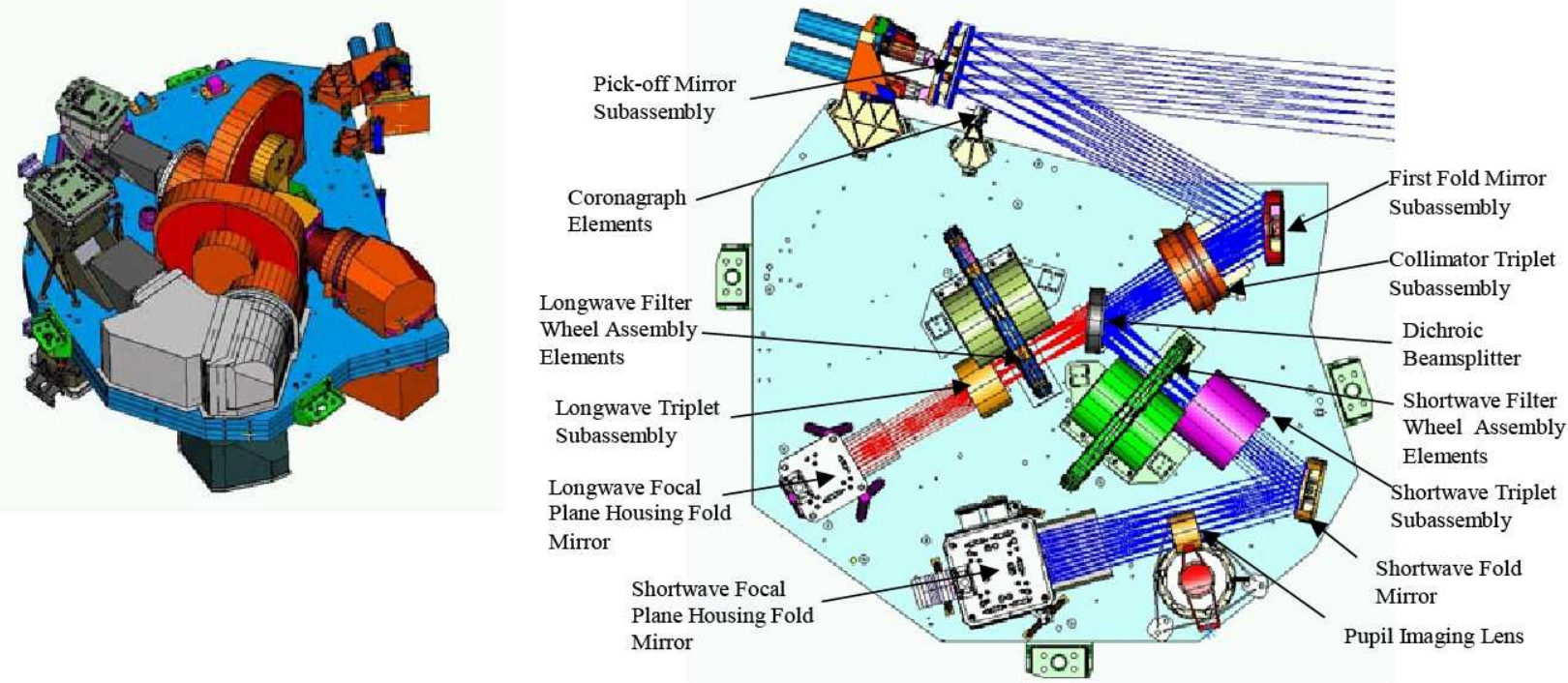

Figure 11: The NIRCam provides high angular resolution imagery

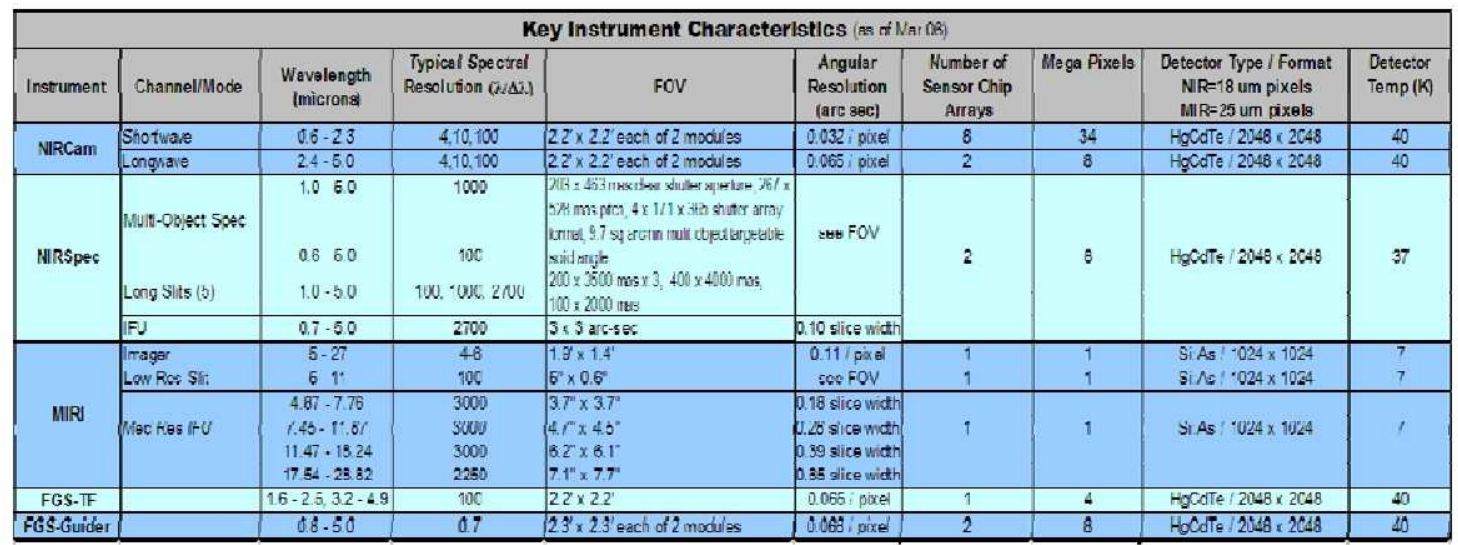

Table 2: JWST science instrument characteristics (coronagraphic modes not shown)

\begin{tabular}{|c|c|c|c|c|c|c|c|}
\hline \multicolumn{8}{|c|}{ JWST Sensitivity } \\
\hline $\begin{array}{l}\text { Wavelength } \\
\text { (microns) }\end{array}$ & Instrument/Mode & Bandwidth (NAX) & SNR & Wall clock Time (s) & $\begin{array}{c}\text { Continuum } \\
\text { Flux Density } \\
\text { (ndy) }\end{array}$ & $\begin{array}{c}\text { Gontinuum } \\
\text { Flux Density } \\
\left(10^{30} \mathrm{~W} \mathrm{~m}^{2} \mathrm{~Hz}^{-1}\right)\end{array}$ & $\begin{array}{l}\text { Unresolved } \\
\text { Line Flux } \\
\left(10^{21} \mathrm{~W} \mathrm{~m}^{2}\right)\end{array}$ \\
\hline 2 & NIRCam & 4 & 10 & 10,000 & 11.40 & 0.11 & NA \\
\hline 3.5 & FGS-TF & 100 & 10 & 10,000 & 126.00 & 1.26 & NA \\
\hline 3 & NIIRSpechow Res & 100 & 10 & 10.000 & 132.00 & 1.32 & NA \\
\hline 2 & MIRSpec/Med Res & NA & 10 & 100,000 & NA & NA & 0.57 \\
\hline 10 & MIRI//Broadband & 5 & 10 & 10,000 & 700.00 & 7.00 & NA \\
\hline 21 & MIRI/Broadband & 4.2 & 10 & 10,000 & 8700.00 & 87.00 & NA \\
\hline 9.2 & MIRI/Spectrometer & 2400 & 10 & 10,000 & NA & NA & 10 \\
\hline 22.5 & MIRI/Spectitometer & 1200 & 10 & 10,000 & NA & NA & 56.00 \\
\hline
\end{tabular}

Table 3: JWST sensitivity benchmarks

Near-infrared scanning Fabry-Perot imagery is provided by the FGS-TF instrument (Doyon 2008). This instrument (Figure 11) provides a narrow-band imaging capability with the same size field of view as the NIRCam and over a similar wavelength range (Table 1). Its applications include deep surveys for emission line objects over a flexible range of redshifts. The instrument includes occulting coronagraphy that can achieve a contrast ratio of $\sim 10^{4}$ using a speckle suppression technique that is enabled by the scanning capability of the etalon. 
The FGS-TF shares an optical bench with the FGS-Guider. The latter is a fully redundant very broad-band camera (Table 1) that functions as the fine guidance sensor for the telescope fine steering mirror system (Figure 7). It delivers guide star centroid measurements with a noise equivalent angle of 3 mas at a rate of $16 \mathrm{~Hz}$. Its wide field of view enables $95 \%$ probability of guide star acquisition over the whole sky and autonomous pattern recognition for guide star identification.
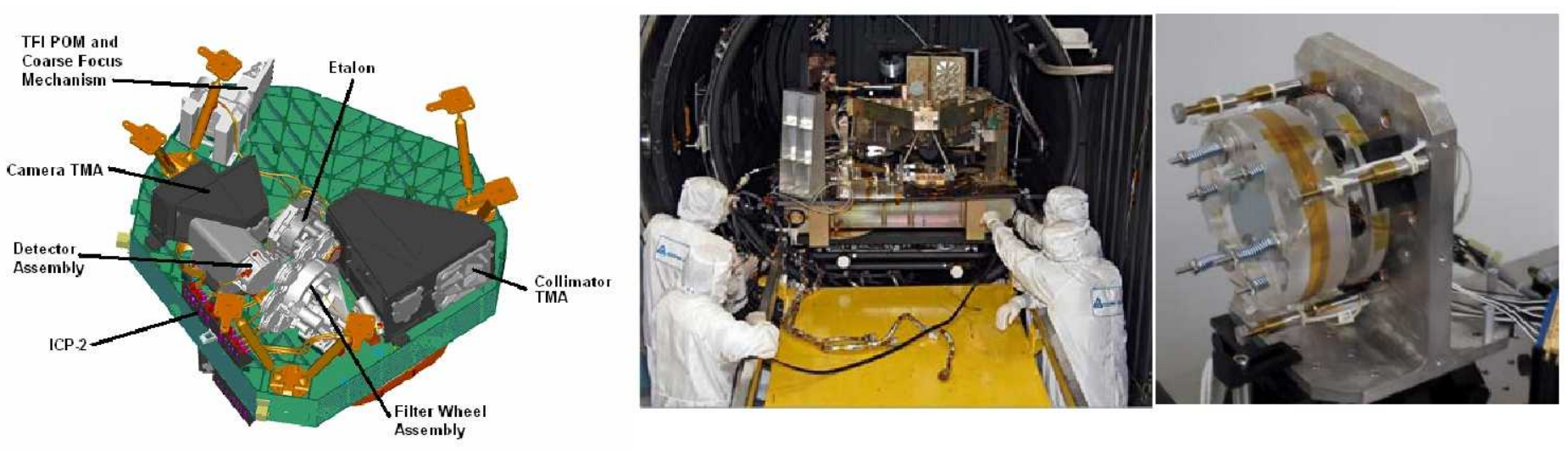

Figure 12: The FGS-TF instrument layout (left), test unit in space simulation chamber (center), and prototype Fabry-Perot etalon (right)

Spectroscopy over the 0.6 - 5 um spectrum is provided by the NIRSpec instrument (Bagnasco 2007). This NIRSpec affords a range of spectral resolutions shown in Table 1 that can be used with long slit, multi-object, and integral field aperture control modes. This instrument (Figure 12) is the first multi-object spectrometer developed for space flight. It is designed to target 100 compact sources simultaneously within a 9 square arcminute field.

Aperture control for multi-object spectroscopy is provided by a $0.25 \mathrm{Mpixel}$ array of micro-shutters (of dimensions shown in Table 1) that are configured for the desired target field based on prior NIRCam imagery. A variety of fixed long slits are provided to enable high contrast and exoplanet transit spectroscopy. Integral field spectroscopy is provided over a 9 square arcsecond field via a conventional image slicer. 


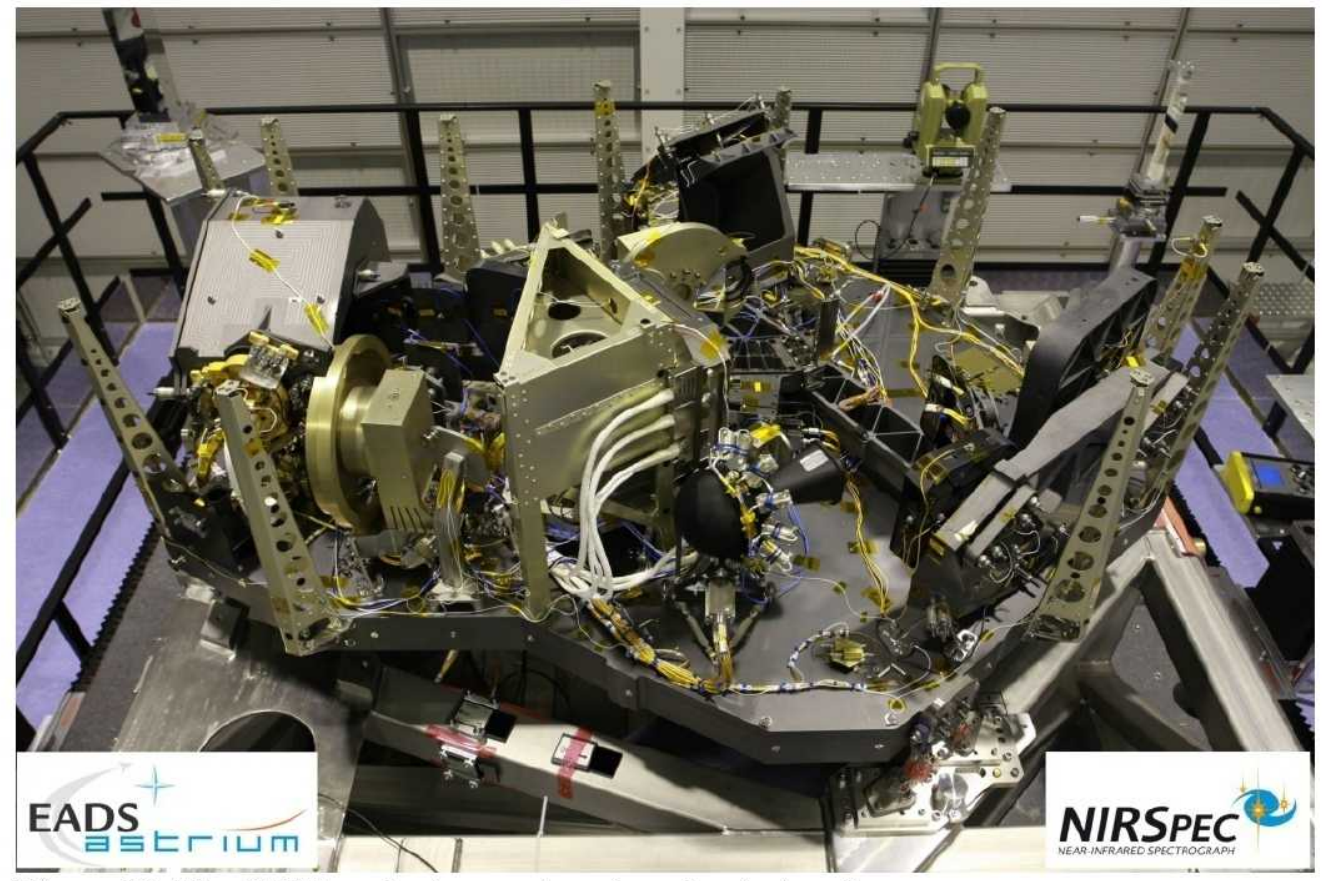

Figure 13: The NIRSpec instrument engineering test unit
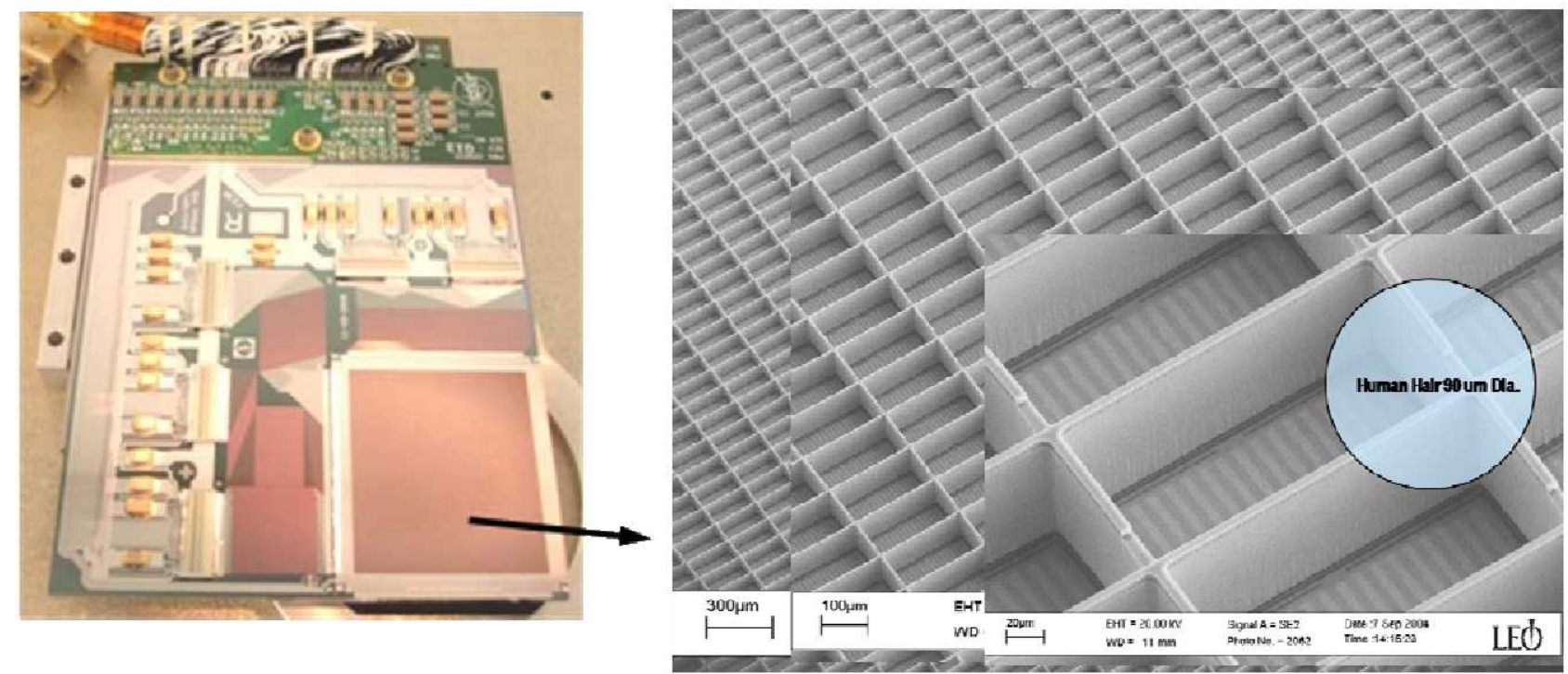

Figure 14: A quadrant of the NIRSpec micro-shutter array for multi-object spectroscopy is shown (left) along with a micrograph of its optical area (right).

Imagery and spectroscopy over the 5-29 um spectrum is provided by the MIRI instrument (Wright 2008). This instrument (Figure 14) provides broad-band imagery, low spectral resolution (1\%) long slit spectroscopy, and medium spectral resolution $\left(\sim 10^{3}\right)$ integral field spectroscopy (Table 1$)$. The imaging mode includes both occulting and quadrant phase mask coronagraphy. The latter type enables very small inner working angle observations of stellar debris disks and exoplanet systems. When used in 
combination with the NIRSpec instrument, an optimally sampled integral field spectrum covering the whole $0.6-29$ um JWST wavelength range can be obtained at medium spectral resolution.
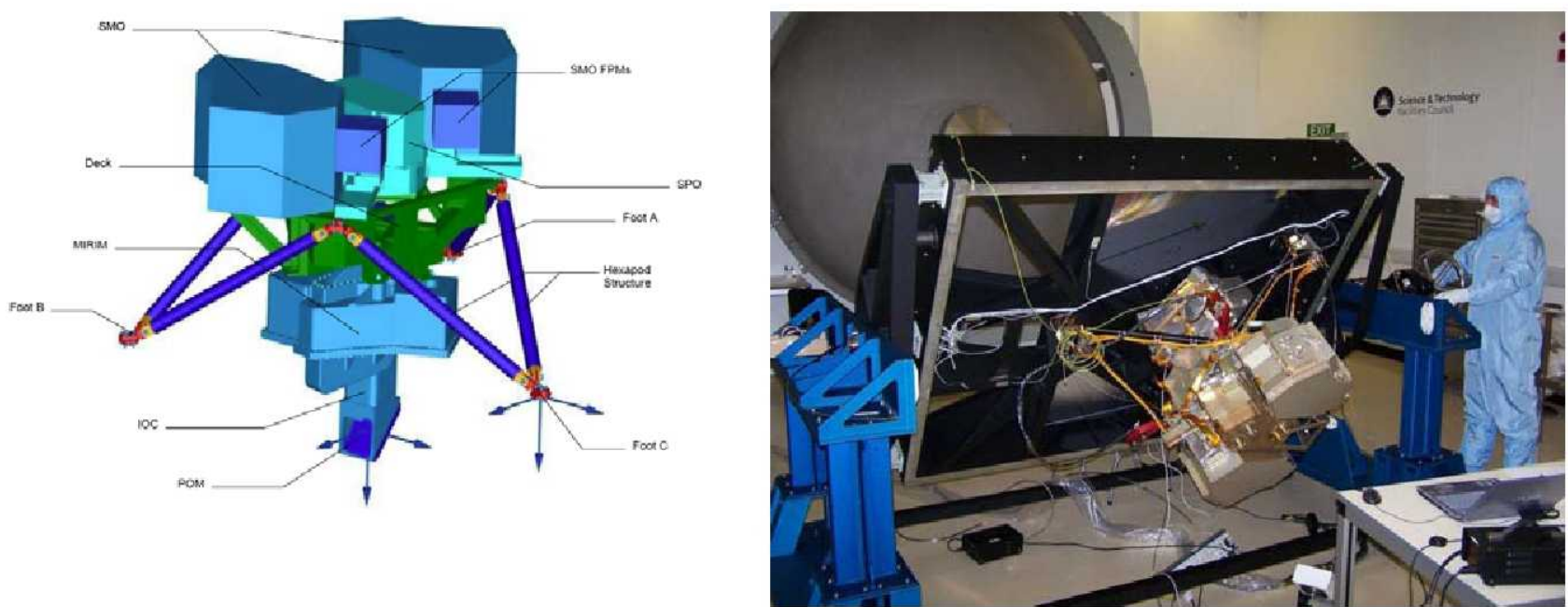

Figure 15: The MIRI instrument configuration (left) and verification model (right)

The detectors for the JWST instruments define the state of art for high performance space flight infrared imaging arrays. The near-infrared instruments utilize $\mathrm{HgCdTe} 4$ Mpixel sensor chip arrays (SCA) operated at $\sim 40 \mathrm{~K}$. Zodiacal background limited sensitivity is achieved in all broad-band instrument modes (Table 3). The MIRI instrument utilizes Si:As 1 Mpixel SCAs operated at $\sim 7 \mathrm{~K}$. Significant gains in noise performance and SCA format were achieved during the JWST technology development phase to enable the above mission science goals (Figures 15, 16). The near-infrared SCAs are designed to be edge-butted on three sides to from larger format focal plane array (FPA) assemblies. For example, the NIRCam short wavelength channel utilizes a 16 Mpixel FPA consisting of 4 of these SCAs, and the NIRSpec utilizes an 8 Mpixel FPA consisting of 2 SCAs. The mid-infrared SCAs are used individually in 1 Mpixel FPA assemblies.
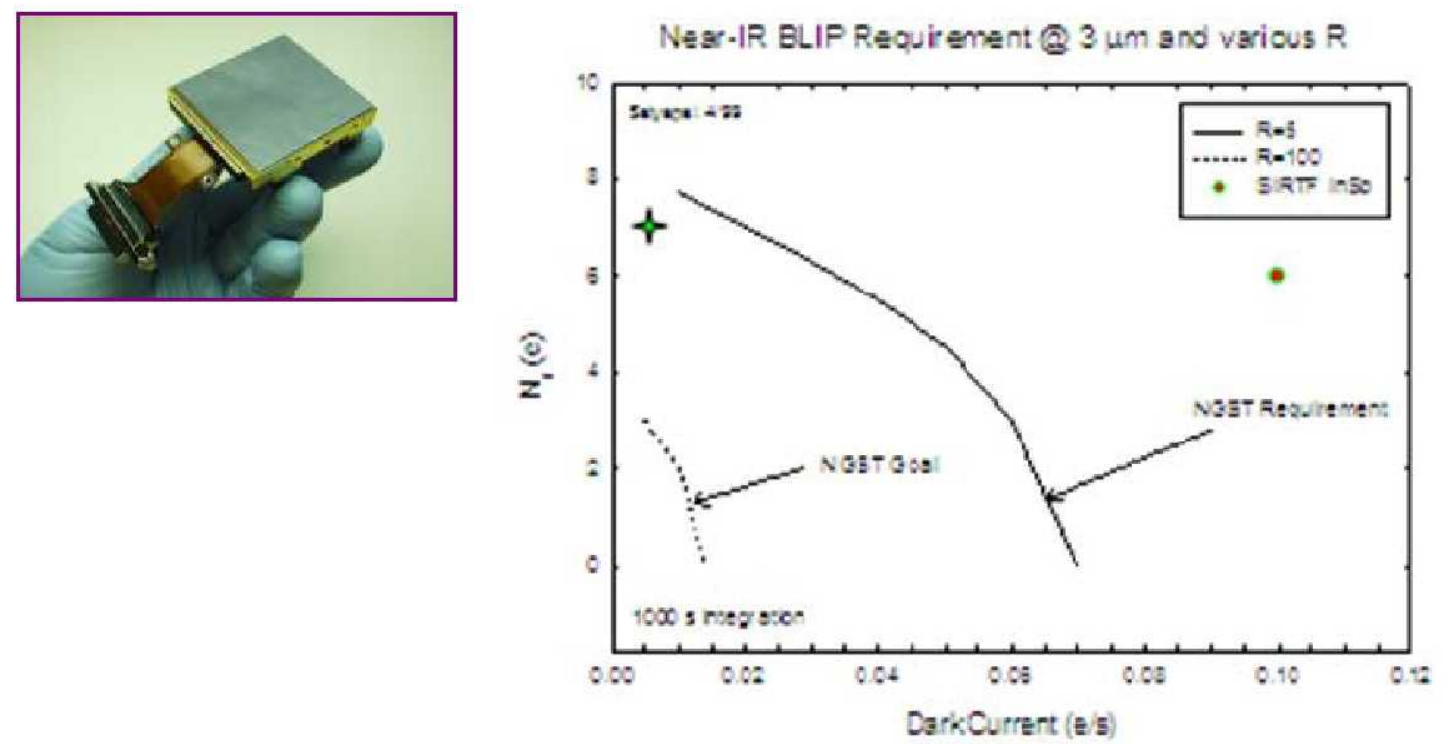

Figure 16: Left -- A Teledyne H2RG 4 Mpixel sensor chip array; Right -- noise performance of this technology (green cross) compared to that of 0.07 Mpixel InSb sensors used on the Spitzer space telescope (red dot). 

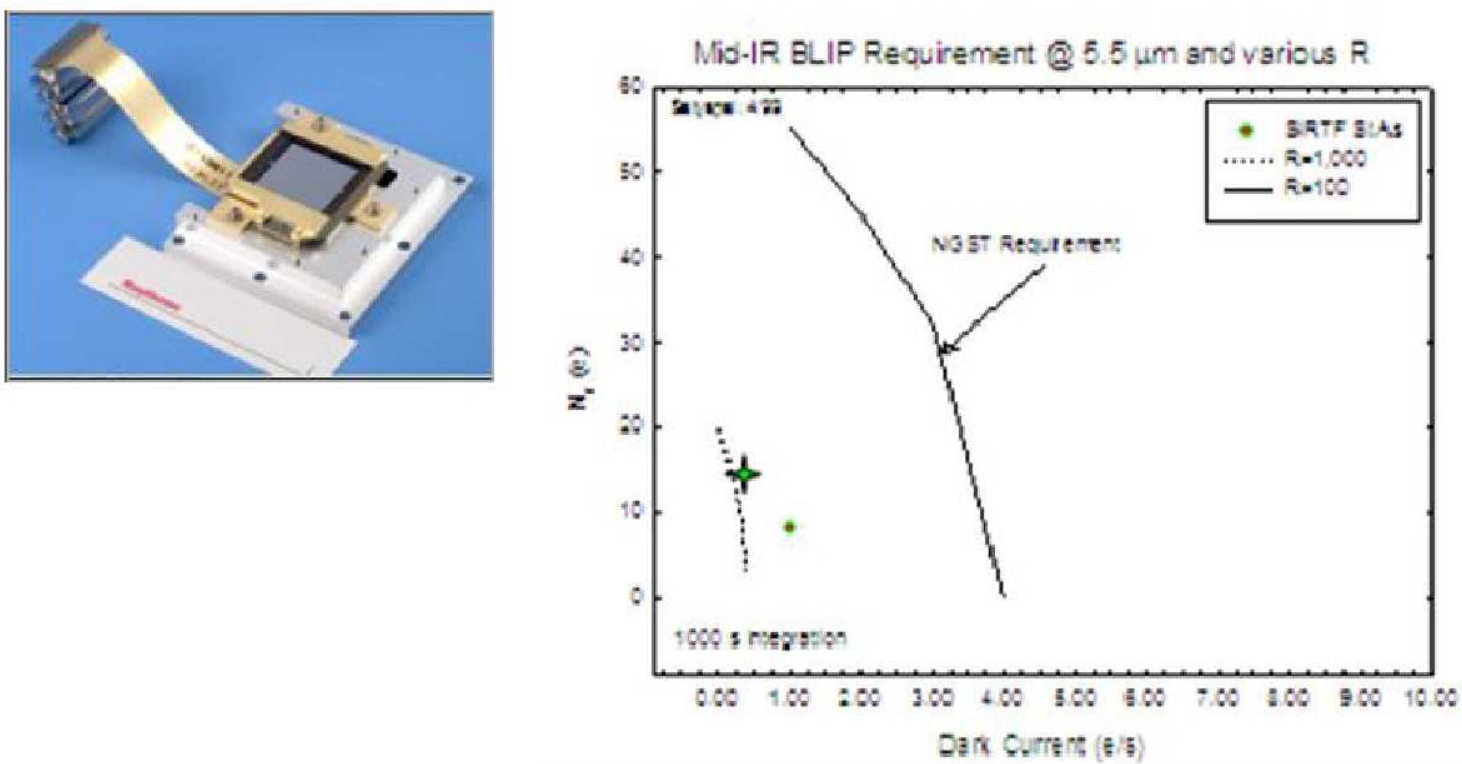

Figure 17: Left -- A Raytheon Si:As 1 Mpixel sensor chip array; Right -- noise performance of this technology (green cross) compared to that of 0.07 Mpixel Si:As sensors used on the Spitzer space telescope (red dot).

\section{Making sure it all works}

Testing at the scale and operating temperature of the JWST requires the largest deep cryogenic $(\sim 30 \mathrm{~K})$ space simulation facilities in the world. The system is built up and tested in incremental steps in which each subunit is tested before it is integrated into the next higher level of assembly. As integration proceeds, larger and larger facilities are needed. The fully integrated instrument payload will be tested at Goddard Space Flight Center using a telescope simulator (Hagopian 2007, Ohl 2009). After integration with the actual telescope, the whole system is tested in a larger facility at Johnson Space Center (Figure 17) (Atkinson 2008). Unlike the Hubble Space telescope, which resides in low earth orbit, the JWST cannot be serviced by astronauts due to its more distant L2 point orbit. However, in contrast to prior space observatories, the JWST telescope and instrument optical systems employ a high degree of freedom for in-flight adjustment (Barto 2008), and lessons learned from prior observatory test programs are carefully taken into account in design of the JWST test program (Feinberg 2008). 

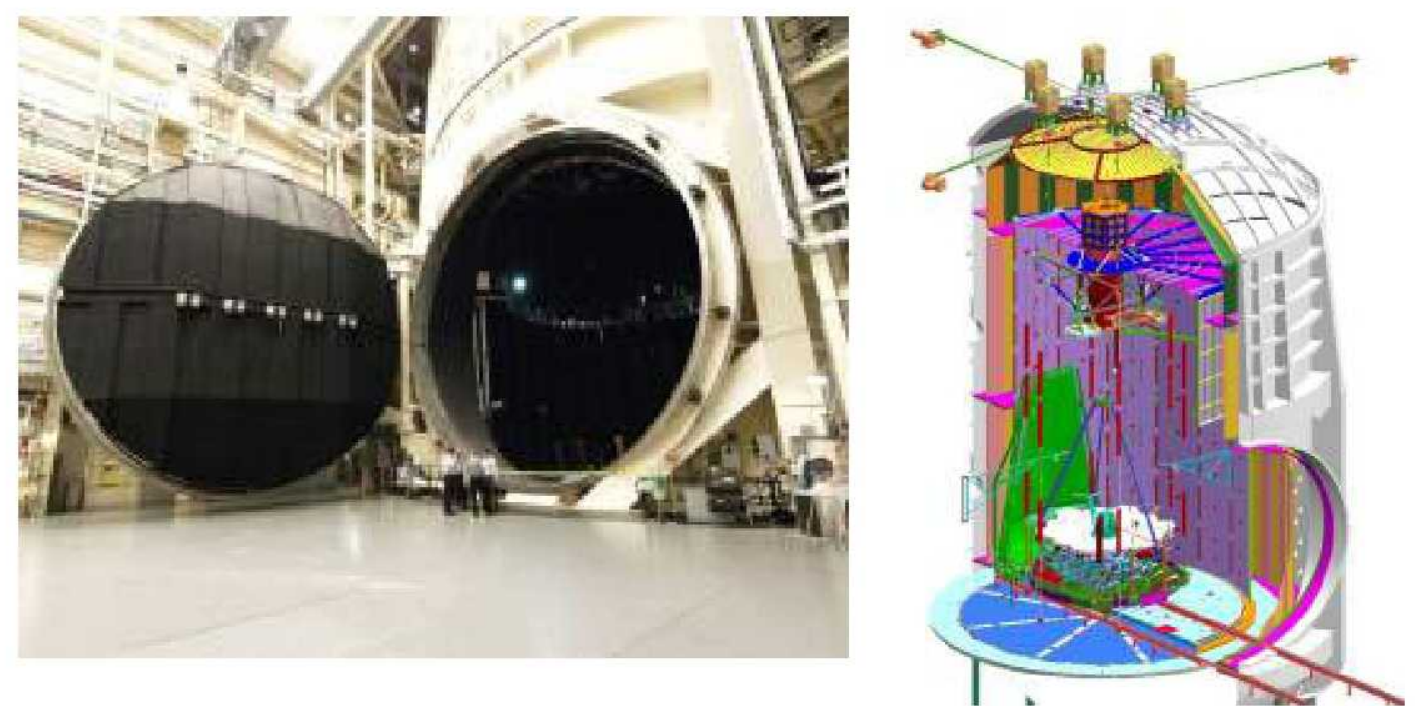

Figure 18: The JWST will be tested in JSC chamber A (120 feet tall, 60 feet diameter)

\section{Synergism with ground-based observatories}

The GTC and future ground-based ELTs will provide unique discovery potential and synergism with the JWST throughout its flight mission and beyond. Ongoing advances in adaptive optics performance toward wide field diffraction limited near-infrared imagery, the inherent ability of ground-based observatories to continuously upgrade science instrumentation, and their overall versatility ensure the power of these facilities at wavelengths where telluric absorption and telescope emission are low.
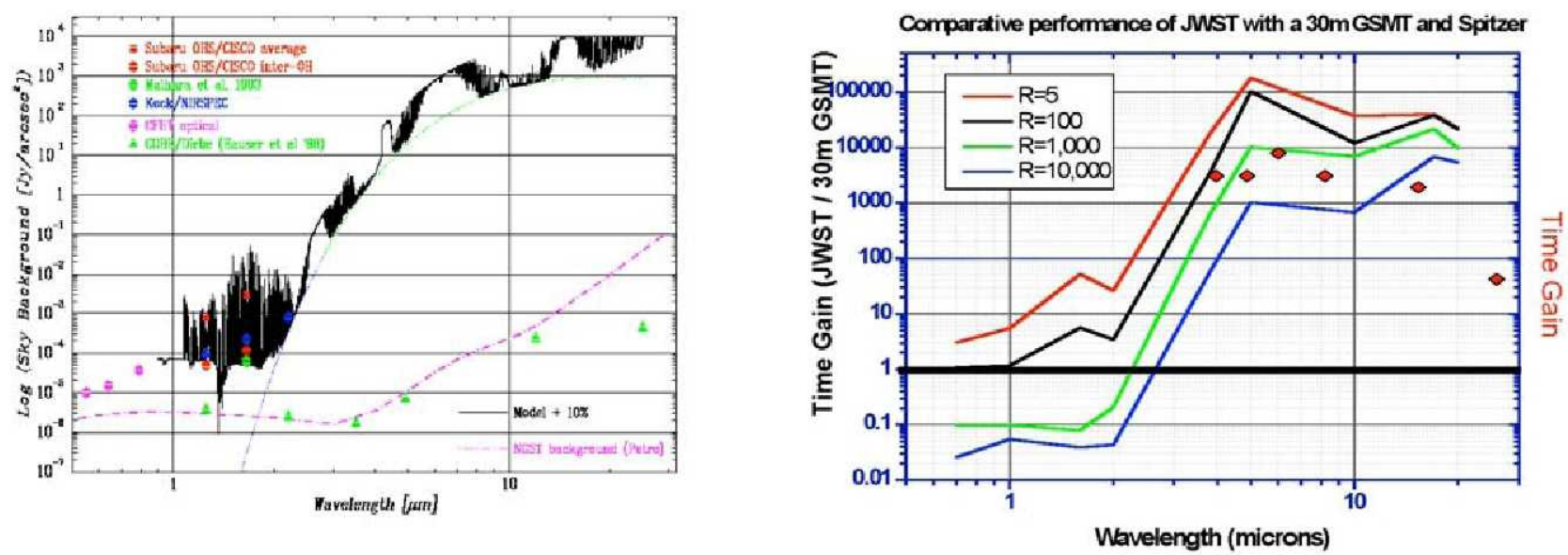

Figure 19: Left - The relative background emission of ground-based and orbital observatories depart markedly at wavelengths $>1.7 \mathrm{um}$; Right -- The relative time gain of JWST compared to a GSMT or Spitzer. The vertical axis is in relative units, where 1.0 means an observation with JWST or GSMT or Spitzer will take the same time to reach equivalent $\mathrm{S} / \mathrm{N}$ on a point source; a number $<1$ means GSMT is faster.

Ground-based and orbital facilities will be powerful partners throughout the JWST era and for the foreseeable future with the former leading in medium and high resolution spectroscopic applications shortward of the thermal infrared (Figure 18). 


\section{References}

Acton, D.S., et al., 2004, "James Webb Space Telescope Wavefront Sensing and Control Algorithms," SPIE, 5487, 877

M. Albanese, A., et al., "Verification of the James Webb Space Telescope coarse phase sensor using the Keck Telescope," Proc. SPIE 6265 (2006)

Atkinson, C. et al., 2008, "Architecting a revised optical test approach for JWST," SPIE, 7010, 24

Bagnasco, G. et al. 2007, "Overview of the near-infrared spectrograph instrument on-board the James Webb Space Telescope," SPIE, 6692, 17

Barto, A., et al., 2008, "Optical performance verification of the James Webb Space Telescope," SPIE, 7010,23

Contos, A., et al., 2008, "Verification of the James Webb Space Telescope (JWST) wavefront sensing and control system," SPIE, 7010, 26

Doyon, R., et al., 2008, “The JWST tunable filter imager (TFI)", SPIE, 7010, 70100

Feinberg, L., et al., 2008 “Applying HST lessons learned to JWST," SPIE, 7010, 13

Feinberg, L., et al., 2007, "TRL-6 for the JWST wavefront sensing and control," SPIE 6687

Gardner, J. P., et al., 2006, Space Sci. Rev., 123, 485

Greenhouse, M. A., et al., 2006, "The James Webb Space Telescope Integrated Science Instrument Module," SPIE 6265

Hagopian, J., et al., 2007 "Optical alignment and test of the James Webb Space Telescope Integrated Science Instrument Module," IEEE Aerospace Conference, 3-10 Mar 2007, Big Sky, Montana

Ohl, R., et al., "Updates to the optical alignment and test plan for the James Webb Space Telescope Integrated Science Instrument Module," SPIE, in press.

Rees, M. J. 1997, in N. R. Tanvir, A. Aragon-Salamanca, \& J. V. Wall eds., The Hubble Space Telescope and the High Redshift Universe, (World Scientific: Singapore), 115

Rieke, M. 2005, " Overview of James Webb Space Telescope and NIRCam's Role," SPIE, 5904, 1

Rieke, M. 2008, see: http://ircamera.as.arizona.edu/nircam/AAS June08.pdf

Wright et al. 2008, "Design and development of MIRI, the mid-IR instrument for JWST," SPIE, 7010, 27 